\title{
ON THE EQUIVALENCE OF EULER-LAGRANGE AND NOETHER EQUATIONS
}

\author{
A. C. FALIAGAS
}

\begin{abstract}
We prove that on the condition of non-trivial solutions, the EulerLagrange and Noether equations are equivalent for the variational problem of nonlinear Poisson equation and a class of more general Lagrangians, including position independent and of $\mathrm{p}$-Laplacian type. As applications we prove certain propositions concerning the nonlinear Poisson equation and its generalisations, the equivalence of admissible and inner variations and discuss the inverse problem of determining the Lagrangian from conservation or symmetry laws.
\end{abstract}

\section{INTRODUCTION}

Alikakos in [1] presented a new method, according to which Derrick-Pohozaev identities and monotonicity formulas can be derived from energy-momentum tensors and reported some interesting applications to the nonlinear Poisson equation. In subsequent work, Alikakos and the author [2, 6] extended these ideas by deriving Derrick-Pohozaev identities and monotonicity formulas in a more systematic fashion and for a larger variety of Lagrangians.

As remarked by the author in [6], Derrick-Pohozaev identities and monotonicity formulas, derived by the aforementioned method, seemed to be more general than those derived by previous methods. More precisely, the starting point for the derivation of Derrick-Pohozaev identity by classical methods is the Euler-Lagrange equations. In the previously mentioned method by Alikakos and the author, however, the Euler-Lagrange equations can be replaced by Noether's equations [9], which is a weaker hypothesis when $u$ is a classical $\mathrm{C}^{2}$ solution ([8], Chapter 3). A simple proof of this fact [8] is provided by Counterexample 1 below.

The foregoing counterexample relies on trivial solutions of Noether's equations. It therefore seemed meaningful to attempt the construction of counterexamples involving non-trivial solutions. In my attempt, I reached the surprising conclusion that, for a rather large class of Lagrangians, only counterexamples involving trivial solutions are possible. In all other cases the Euler-Lagrange and Noether's equations are equivalent. The proof of this statement is the main result of this paper. Theorem 1 proves for the Lagrangian of the nonlinear Poisson equation that every non-trivial classical solution of Noether's system is necessarily a solution of the

I would like to express my thanks to N. Alikakos for reading this paper and suggesting many useful improvements. 
Euler-Lagrange equation. Theorem 2 states a condition under which the EulerLagrange and Noether's equations are equivalent. Theorems 3 and 4 extend the basic idea to more general Lagrangians. In Section 2 there is a review of inner variations, energy-momentum tensors and other prerequisite material, serving mainly to introduce notations. More details on these topics are found in [8, 6].

Finally, there is yet another reason for considering the undertaking of this research meaningful. According to Noether's theorem, given any Lagrangian, there is a conservation law corresponding to each continuous "symmetry transformation", as are called in the physical literature transformations leaving the Lagrangian and the equations of motion invariant in form ([7], ï œ $œ 52-3)$. This permits observed selection rules in nature to be directly transposed into symmetry requirements on the Lagrangian and is used by physicists as a guide for the introduction of interaction terms when developing new Lagrangians in quantum field theory ([3], ï œ11.4, p. 17). But if we know that a set of conservation laws is equivalent to the equations of motion (i.e. the Euler-Lagrange equations), this should already determine the Lagrangian or at least a class of equivalent Lagrangians. We demonstrate this by an example in Section 5

In this paper, we are concerned with the purely technical mathematical-analytical aspects of the subject, leaving the applications to physics for subsequent work.

\section{NOETHER's EQuATIONS}

In this section we summarise background material which is necessary for the understanding of the statement and proof of main results and serves as a means for the introduction of notations to be used. The standard reference is [8].

2.1. General notation. Throughout this paper $\Omega$ is a domain (open connected subset) of $\mathbb{R}^{N}$, except when otherwise stated. The following abbreviated notation

$$
u_{, i}=\frac{\partial u}{\partial x_{i}}
$$

is used for partial derivatives. Einstein's summation convention applies everywhere, except when the contrary is explicitly mentioned.

Following standard notation, $\mathrm{C}^{r}(\Omega)$ is the set of $r$ times continuously differentiable functions in $\Omega$ and $\mathrm{C}^{r}(\bar{\Omega})$ the set of restrictions to $\Omega$ of $r$ times continuously differentiable functions in $\mathbb{R}^{N}$. The set of $r$ times continuously differentiable, $\mathbb{R}^{M}$ (or $\mathbb{C}^{M}$ ) valued functions in $\Omega$ is denoted by $C^{r}(\Omega)^{M}$ and the corresponding set of restrictions to $\Omega$ of $r$ times continuously differentiable functions in $\mathbb{R}^{N}, \mathrm{C}^{r}(\bar{\Omega})^{M}$. $\mathscr{D}(\Omega)$ denotes the set of real (or complex) $\mathrm{C}^{\infty}$ functions on $\Omega$ with compact support in $\Omega$ and $\mathscr{D}(\Omega)^{M}$ the set of $\mathbb{R}^{M}$ (or $\mathbb{C}^{M}$ ) valued $\mathrm{C}^{\infty}$ functions on $\Omega$ with compact support in $\Omega$.

2.2. Variational functionals. We will be considering (nonlinear) functionals $J$ : $\mathrm{C}^{1}(\bar{\Omega})^{M} \rightarrow \mathbb{R}, M \in \mathbb{N}$, of the form

$$
J(u):=\int_{\Omega} L(x, u(x), D u(x)) d x,
$$


where $\Omega$ is a bounded domain of $\mathbb{R}^{N}, L(x, y, z)$ a Lagrangian,

$$
L: \Omega \times \mathbb{R}^{M} \times \mathbb{R}^{N \cdot M} \rightarrow \mathbb{R}
$$

$L \in \mathrm{C}^{1}\left(\bar{\Omega} \times \mathbb{R}^{M} \times \mathbb{R}^{N \cdot M}\right)$ and $u \in \mathrm{C}^{1}(\bar{\Omega})^{M}$.

A function $u \in \mathrm{C}^{1}(\Omega)^{M}$ is a critical point of $J$ when

$$
\delta J(u) v:=\left.\frac{d}{d t} J(u+t v)\right|_{t=0}=0 \quad \forall v \in \mathscr{D}(\Omega)^{N} .
$$

The derivative $\delta J(u) v$ is the variation of $J$ at $u$ in direction $v$. When $u \in \mathrm{C}^{2}(\Omega)^{M}$, an easy calculation shows

$$
\delta J(u) v=\int_{\Omega} \delta L(u) \cdot v d x
$$

where $\delta L(u)=\left(\delta L(u)_{i}\right)_{i=1, \cdots, M}$ is the vector field with components

$$
\delta L(u)_{i}=\left.\left(L_{y_{i}}-\frac{\partial}{\partial x_{j}} L_{z_{i j}}\right)\right|_{(x, u(x), D u(x))} .
$$

We will refer to $\delta L$ as the Euler-Lagrange derivative. Every critical point $u \in$ $\mathrm{C}^{2}(\Omega)^{M}$ of $J$ satisfies the Euler-Lagrange equations

$$
\delta L(u)=0 .
$$

2.3. Inner variations. Inner variations are a special kind of variations. Let $\mathrm{I}=$ ]$-\delta, \delta\left[, \delta>0\right.$. Fixing a $u \in \mathrm{C}^{1}(\Omega)^{M}$ and a set of diffeomorphisms $\left(\xi^{t}\right)_{t \in \mathrm{I}}, \xi^{t}$ : $\Omega \rightarrow \Omega$, the following set of functions

$$
\widetilde{u}(x, t):=u\left(\xi^{t}(x)\right), \quad t \in \mathrm{I}
$$

define a set of variations of $u$ under certain conditions, to be made precise in the following definition.

Definition 1. A) Let $h \in \mathscr{D}(\Omega)^{N}, \delta>0$ and I $\left.=\right]-\delta, \delta$ [. A set of diffeomorphisms $\left(\xi^{t}\right)_{t \in \mathrm{I}}$ of $\Omega$ having the properties (i) - (iii) below and such that the function $\xi$ : $\Omega \times \mathrm{I} \rightarrow \Omega, \xi(x, t)=\xi^{t}(x)$ is $\mathrm{C}^{\infty}$-differentiable, is called an inner variation of $\Omega$ in direction $h$ or which is defined by $h$ :

(i) $\xi^{0}=i d_{\Omega}$, i.e. $\xi^{0}(x)=x$ in $\Omega$.

(ii) $D_{t} \xi(x, 0)=h(x) \forall x \in \Omega$.

(iii) $\xi^{t} \mid \partial \Omega=i d_{\Omega}$, i.e. $\xi^{t}(x)=x \forall x \in \partial \Omega$.

B) Let $J$ be a functional satisfying (VF), $u \in \mathrm{C}^{1}(\bar{\Omega})^{M}, h \in \mathscr{D}(\Omega)^{N}$ and $\left(\xi_{h}^{t}\right)_{t \in \mathrm{I}}$ the inner variation of $\Omega$ defined by $h$. The set of functions

$$
u \circ \xi_{h}^{t}, \quad t \in \mathrm{I}
$$

is called the inner variation of $u$ in direction $h$. The derivative

$$
\mathfrak{o} J(u) h:=\left.\frac{d}{d t} J\left(u \circ \xi_{h}^{t}\right)\right|_{t=0},
$$

is called the inner variation of the functional $J$ at $u$ in direction $h$.

For the calculation of inner variations the following proposition is used. 
Proposition 1. Let $J$ be a functional satisfying (VF) and $u \in \mathrm{C}^{1}(\bar{\Omega})^{M}$. The inner variation of $J$ at $u$ is given by

$$
\mathfrak{d} J(u) h=\int_{\Omega}\left(u_{k, i} L_{z_{k j}} h_{i, j}-L \operatorname{div} h-L_{x_{i}} h_{i}\right) d x, \quad h \in \mathscr{D}(\Omega)^{N},
$$

where $L, L_{x_{i}}:=\frac{\partial L}{\partial x_{i}}, L_{z_{k j}}:=\frac{\partial L}{\partial z_{k j}}$ are taken at the point $(x, u(x), D u(x))$, i.e. $L=$ $L(x, u(x), D u(x)), L_{x_{i}}=L_{x_{i}}(x, u(x), D u(x))$ etc.

Proof. The proof consists in considering the function $\varphi(t):=J\left(u \circ \xi^{t}\right)$, applying the change of integration variable $x=\eta^{t}(y)$, where $\eta^{t}$ is the inverse function of $\xi^{t}$, differentiating with respect to $t$ and interchanging differentiation with integration. The details are lengthy and have been omitted as they are available in [6].

\subsection{Energy-momentum tensor. On using the notation}

$$
\mathfrak{d} L(u) h:=u_{k, i} L_{z_{k j}}(\cdot, u, D u) h_{i, j}-L(\cdot, u, D u) h_{i, i}-L_{x_{i}}(\cdot, u, D u) h_{i}
$$

the expression for the inner variation of $J$ reduces to

$$
\mathfrak{d} J(u) h=\int_{\Omega} \mathfrak{d} L(u) h d x, \quad h \in \mathscr{D}(\Omega)^{N} .
$$

When $L_{x}=0$, formula (4) simplifies further to

$$
\mathfrak{d} L(u) h:=u_{k, i} L_{z k j} h_{i, j}-L \delta_{i j} h_{i, j}=\left(u_{k, i} L_{z k j}-L \delta_{i j}\right) h_{i, j} .
$$

This motivates the following definition of the energy-momentum tensor.

Definition 2. Let $J$ be a functional satisfying (VF). The energy-momentum tensor of the variational problem specified by $J$, is defined by

$$
T_{i j}(x, y, z)=z_{k i} L_{z_{k j}}(x, y, z)-\delta_{i j} L(x, y, z)
$$

where $x=\left(x_{i}\right)_{i=1, \cdots, N} \in \Omega, y=\left(y_{k}\right)_{k=1, \cdots, M} \in \mathbb{R}^{M}, z=\left(z_{k i}\right)_{k=1, \cdots, M ; i=1, \cdots, N} \in \mathbb{R}^{N M}$.

Remark 1. Notice that the above definition holds for general variables $(x, y, z) \in$ $\Omega \times \mathbb{R}^{M} \times \mathbb{R}^{N M}$, not just for $(x, u(x), D u(x)), x \in \Omega$. Given any vector field $u \in$ $\mathrm{C}^{1}(\Omega)^{M}$ we have the tensor field

(7) $T_{i j}(x)=T_{i j}(x, u(x), D u(x))=u_{k, i} L_{z_{k j}}(x, u(x), D u(x))-\delta_{i j} L(x, u(x), D u(x))$,

for which we will be using the same symbol. Again, in this formula it is not necessary that $u$ be a solution of the Euler-Lagrange equations.

2.5. Noether's equations. Let $u \in \mathrm{C}^{2}(\Omega)$, which is not necessarily a solution of the Euler-Lagrange equations and $T_{i j}(x)=T_{i j}(x, u(x), D u(x))$. By the definition of energy-momentum tensor

$$
\begin{aligned}
T_{i j, j} & =\frac{\partial}{\partial x_{j}}\left(u_{k, i} L_{z_{k j}}-\delta_{i j} L\right) \\
& =u_{k, i j} L_{z_{k j}}+u_{k, i} \frac{\partial}{\partial x_{j}} L_{z_{k j}}-L_{x_{i}}-L_{y_{k}} u_{k, i}-L_{z_{k j}} u_{k, i j} \\
& =\left(\frac{\partial}{\partial x_{j}} L_{z_{k j}}-L_{y_{k}}\right) u_{k, i}-L_{x_{i}}
\end{aligned}
$$


where $L=L(x, u(x), D u(x)), L_{y_{k}}=L_{y_{k}}(x, u(x), D u(x))$ and $L_{z_{k j}}=L_{z_{k j}}(x, u(x), D u(x))$. From this we obtain

$$
T_{i j, j}+L_{x_{i}}=\left(\frac{\partial}{\partial x_{j}} L_{z_{k j}}-L_{y_{k}}\right) u_{k, i}
$$

which motivates the following definition.

Definition 3. The system of second order partial differential equations

$$
T_{i j, j}(x, u(x), D u(x))+L_{x_{i}}(x, u(x), D u(x))=0
$$

or in index-free notation

$$
\operatorname{div} T(x, u(x), D u(x))+L_{x}(x, u(x), D u(x))=0
$$

is called Noether's equations.

If we define inner critical points of $J$ by $\mathfrak{d} J(u)=0$, i.e. $\mathfrak{d} J(u) h=0 \forall h \in \mathscr{D}(\Omega)^{N}$, then Noether's equations are related to inner critical points in an analogous manner as critical points to the Euler-Lagrange equations. Furthermore, by (8) every solution $u \in \mathrm{C}^{2}(\Omega)$ of the Euler-Lagrange equations is a solution of Noether's equations. The converse of this statement is in general not true. Giaquinta and Hildebrandt [8] presented the following simple counterexample to demonstrate this.

Counterexample 1. Let $F \in \mathrm{C}^{1}(\mathbb{R}), F \neq$ const. and

$$
J(u):=\int_{\Omega} F(u(x)) d x, \quad u \in \mathrm{C}^{2}(\Omega) \cap \mathrm{C}^{1}(\bar{\Omega}) .
$$

The energy-momentum tensor is calculated by (6)

$$
T=-F(u) I
$$

and Noether's equations (9) reduce to

$$
F^{\prime}(u) D u=0 .
$$

It is obvious that every constant function $u=c_{0}$ is a solution of this system, but not of the Euler-Lagrange equations, which for this functional assume the form

$$
F^{\prime}(u)=0 \text {. }
$$

We will show in the next two sections that only trivial counterexamples are possible for a large class of Lagrangians.

\section{Nonlinear Poisson Equation}

In this section we present the main theorem for the nonlinear Poisson equation, see equation (12) below, in a bounded domain $\Omega$, which can be viewed as the EulerLagrange equation of a variational functional $J$ with the Lagrangian

$$
L(u, z)=\frac{1}{2}|z|^{2}+F(u),
$$

where $z$ corresponds to $D u$ when $u$ is a $C^{1}$ function and $F: \mathbb{R} \rightarrow \mathbb{R}$. When $F \in$ $\mathrm{C}^{1}(\mathbb{R}), J$ clearly conforms to requirements (VF). 
Theorem 1. Let $\Omega$ be a bounded domain of $\mathbb{R}^{N}, F \in \mathrm{C}^{1}(\mathbb{R})$, L a Lagrangian of the form (10) and $u \in \mathrm{C}^{2}(\Omega) \cap \mathrm{C}^{1}(\bar{\Omega})$ a non-trivial classical solution of Noether's equations

$$
\operatorname{div} T(u, D u)=0 .
$$

where $T$ is the energy-momentum tensor corresponding to the Lagrangian $L$ with components $T_{i j}=u_{, i} u_{, j}-\delta_{i j} L$. Then $u$ is a solution of the Euler-Lagrange equation

$$
\Delta u=F^{\prime}(u)
$$

in $\Omega$.

Remark 2. By (8), equation (11) is equivalently written in the form

$$
\left(\Delta u-F^{\prime}(u)\right) D u=0 .
$$

The non-triviality condition $D u \neq 0$ means $D u$ is not identically 0 , i.e. there is a $x_{0} \in \Omega$ such that $D u\left(x_{0}\right) \neq 0$.

Proof. Let $u$ be a solution of (11) and set

$$
\mathrm{A}_{0}:=\{x \in \Omega: D u(x)=0\}
$$

and

$$
\mathrm{A}_{1}:=\{x \in \Omega: D u(x) \neq 0\} .
$$

Obviously $\mathrm{A}_{0}$ is closed relatively $\Omega, \mathrm{A}_{1}$ is open and $\mathrm{A}_{0} \cup \mathrm{A}_{1}=\Omega$. It is clear that (12) is satisfied in $A_{1}$. We have to show (12) is also satisfied in $A_{0}$.

Step 1. Let $\mathrm{D}$ be the subset of $\Omega$ in which the Euler-Lagrange equation (12) is satisfied, i.e.

$$
\mathrm{D}:=\{x \in \Omega: \Delta u(x)=f(u(x))\},
$$

where $f:=F^{\prime}$. D is obviously closed relatively $\Omega$ and we have already shown that

$$
\mathrm{A}_{1} \subset \mathrm{D} \text {. }
$$

From this, keeping in mind that closures and frontiers are taken relatively $\Omega$, it follows immediately that $\overline{\mathrm{A}}_{1} \subset \mathrm{D}$, hence also

$$
\partial \mathrm{A}_{1} \subset \mathrm{D} \text {. }
$$

It is our intention to show that

$$
\partial \mathrm{A}_{0} \subset \mathrm{D} .
$$

For this purpose we will show $\partial \mathrm{A}_{0}=\partial \mathrm{A}_{1}$, from which (14) follows immediately. Indeed, from $\partial \mathrm{A}_{0}=\mathrm{A}_{0} \backslash \stackrel{\circ}{\mathrm{A}}_{0}$ and

$$
\partial \mathrm{A}_{1}=\overline{\mathrm{A}}_{1} \backslash \stackrel{\circ}{\mathrm{A}}_{1}=\left(\Omega \backslash \stackrel{\circ}{\mathrm{A}}_{0}\right) \backslash\left(\Omega \backslash \mathrm{A}_{0}\right)=\left(\Omega \backslash \stackrel{\circ}{\mathrm{A}}_{0}\right) \cap \mathrm{A}_{0}=\mathrm{A}_{0} \backslash \stackrel{\circ}{\mathrm{A}}_{0}
$$

we get $\partial \mathrm{A}_{0}=\partial \mathrm{A}_{1}$ and with this the validity of (14).

If ${\stackrel{\circ}{\mathrm{A}_{0}}}=\emptyset$ we are finished, for $\mathrm{A}_{0}=\partial \mathrm{A}_{0} \subset \mathrm{D}$. Let $\stackrel{\circ}{\mathrm{A}}_{0} \neq \emptyset$. By hypothesis, for all $x \in \AA_{0}$ we have $D u(x)=0$, hence also $D^{2} u(x)=0$ on $\AA_{0}$ and $u(x)=$ const. on connected components of $\mathrm{A}_{0}$. 
Step 2. Fix $x_{0} \in \stackrel{\circ}{\mathrm{A}}_{0}$. We will show that there is a $x_{1} \in \partial \mathrm{A}_{0}$ and a continuous curve $\left.\gamma: \overline{\mathrm{I}} \rightarrow \mathrm{A}_{0}, \mathrm{I}=\right] 0,1\left[\right.$, such that $\gamma(0)=x_{0}, \gamma(1)=x_{1}$ and $\gamma\left(\left[0,1[) \subset \stackrel{\circ}{\AA}_{0}\right.\right.$, i.e. the curve lies in the interior of $\mathrm{A}_{0}$, with the exception of $x_{1}$. Let $y \in \mathrm{A}_{1} \neq \emptyset$ by hypothesis and $\alpha: \overline{\mathrm{I}} \rightarrow \Omega$ a continuous curve connecting $x_{0}=\alpha(0)$ and $y=\alpha(1)$. The set

$$
\Gamma:=\left\{\alpha(t): t \in \overline{\mathrm{I}}, \alpha(t) \in \partial \mathrm{A}_{0}\right\}
$$

is not empty ([5], (3.19.9) and following Remark, p. 70). Since $\{t \in \overline{\mathrm{I}}: \alpha(t) \in$ $\left.\partial \mathrm{A}_{0}\right\}=\alpha^{-1}\left(\partial \mathrm{A}_{0}\right)$ is closed, $\tau:=\inf \left\{t \in \overline{\mathrm{I}}: \alpha(t) \in \partial \mathrm{A}_{0}\right\} \in \alpha^{-1}\left(\partial \mathrm{A}_{0}\right)$, hence $x_{1}:=$ $\alpha(\tau) \in \partial \mathrm{A}_{0}$ and it is clear that $\alpha\left(\left[0, \tau[) \subset \AA_{0}\right.\right.$. For if there were a $\tau_{1}<\tau$ such that $y^{\prime}=\alpha\left(\tau_{1}\right) \notin \stackrel{\circ}{\mathrm{A}}_{0}$, then $y^{\prime} \notin \mathrm{A}_{0}$ and application of the same procedure for $x_{0}, y^{\prime} \in \mathrm{A}_{1}$ would yield the existence of a $x_{1}^{\prime}=\alpha\left(\tau^{\prime}\right) \in \partial \mathrm{A}_{0}$ with $\tau^{\prime}<\tau$, which contradicts the definition of $\tau$. Reparametrisation of $\alpha \mid[0, \tau]$ yields $\gamma$.

Step 3. Now let $u\left(x_{0}\right)=: c_{0}$. Since $x_{0}$ and $x_{1}$ belong to the same connected component of $\mathrm{A}_{0}$, we have $u\left(x_{1}\right)=c_{0}$ and $f\left(u\left(x_{0}\right)\right)=f\left(u\left(x_{1}\right)\right)=f\left(c_{0}\right)=: d_{0}$. Since by (14) $x_{1} \in \mathrm{D}$, we have

$$
\Delta u\left(x_{1}\right)=f\left(u\left(x_{1}\right)\right)=d_{0} .
$$

But

$$
\Delta u\left(x_{1}\right)=\Delta u\left(\lim _{t \rightarrow 1-} \gamma(t)\right)=\lim _{t \rightarrow 1-} \Delta u(\gamma(t))=0
$$

for $\gamma(t) \in \AA_{0}$ for all $t \in\left[0,1\left[\right.\right.$. Combination of (15) and (16) yields $d_{0}=0$, hence

$$
f\left(u\left(x_{0}\right)\right)=0 .
$$

This means in particular

$$
\Delta u\left(x_{0}\right)-f\left(u\left(x_{0}\right)\right)=0 .
$$

With this we have proved $\AA_{0} \subset \mathrm{D}$ and by (14) $\mathrm{A}_{0} \subset \mathrm{D}$.

From the proof of this theorem we conclude without difficulty the following Corollary.

Corollary 1. Under the assumptions of Theorem $\square$ if the set $\mathrm{A}_{0}:=\{x \in \Omega$ : $D u(x)=0\}$ has an interior point, then $f(u)=0$ on all of $\AA_{0}$.

Proof. By hypothesis $\mathrm{A}_{1} \neq \emptyset$. Let $x_{0} \in \stackrel{\circ}{\mathrm{A}}_{0}$. Now Steps 2 and 3 of the proof of Theorem 11apply and from (17) it follows that $f\left(u\left(x_{0}\right)\right)=0$. Since $x_{0}$ was arbitrary, the assertion is proved.

As an application, we state the following result for the nonlinear Poisson equation.

Corollary 2. Let $\Omega$ be a bounded domain of $\mathbb{R}^{N}$ and $f \in \mathrm{C}(\mathbb{R})$ such that $f(t) \neq 0$ for all $t \in \mathbb{R}$. Then for every solution $u \in \mathrm{C}^{2}(\Omega) \cap \mathrm{C}^{1}(\bar{\Omega})$ of the nonlinear Poisson equation

$$
\Delta u=f(u)
$$

the set $\mathrm{A}_{0}:=\{x \in \Omega: D u(x)=0\}$ has no interior point. 
Proof. Equation (18) is the Euler-Lagrange equation of the functional (10) with $F(t)=\int_{0}^{t} f(s) d s$, for which we have $F \in \mathrm{C}^{1}(\mathbb{R})$. If $\AA_{0} \neq \emptyset$, by Corollary 1 we would have $f(u)=0$ on $\stackrel{\circ}{\mathrm{A}}_{0}$, which is absurd.

Remark 3. In a too strict sense, Noether's equations would never be equivalent to the Euler-Lagrange equations, if $F^{\prime}(c) \neq 0$ for some $c \in \mathbb{R}$. For by Remark 2 constant functions are always solutions of Noether's equations, and then the assumption of equivalence would lead to $F^{\prime}(c)=0$, by considering the constant function $u=c$, which as said is a solution of Noether's equations. Aside from the fact that constant solutions are of little practical importance, since, taking the example of quantum theory, they may represent only special instances of physical states, or they might be not integrable when $\Omega$ is not bounded; they never occur when one considers non-constant boundary conditions. Equivalence of Euler-Lagrange and Noether's equations is achieved, if one directly excludes constant solutions or imposes additional hypotheses such as boundary conditions as in the following Theorem.

Theorem 2. Let $\Omega$ be a bounded domain of $\mathbb{R}^{N}, F \in \mathrm{C}^{1}(\mathbb{R}), g \in \mathrm{C}(\partial \Omega)$ a nonconstant function and $\mathscr{H}:=\left\{u \in \mathrm{C}^{2}(\Omega) \cap \mathrm{C}^{1}(\bar{\Omega}): u \mid \partial \Omega=g\right\}$. Then the EulerLagrange and Noether equations for the Lagrangian (10) are equivalent in $\mathscr{H}$.

Proof. It follows immediately by Theorem 1 for every $u \in \mathscr{H}$ is non-trivial.

Remark 4. We have restricted the above discussion to bounded domains only for the sake of convenience. Indeed, this hypothesis serves to maintain integrability in (VF) and guarantee the exchange of differentiation and integration. The same purpose also serves the hypothesis $u \in \mathrm{C}^{1}(\bar{\Omega})$ with the exception of Theorem 2 . Thus the above results, with proper modifications, are applicable to unbounded domains as well.

\section{More General Lagrangians}

We proceed to generalizing the results of the previous section by considering Lagrangians of the form $L(x, u, z)$, which, along with (VF), satisfy the condition $(\mathrm{H})$ below. Again, $u$ is a scalar function and argument $z$ corresponds to $\nabla u$.

$$
\begin{aligned}
& L_{x_{i} z_{i}}(x, u, 0)=0 \text { for all } x, u . \\
& L_{x_{i} u}(x, u, 0)=0 \text { for all } x, u \text { and all } i=1, \cdots, N .
\end{aligned}
$$

Example 1. (i) All Lagrangians which are independent of $x$ satisfy $(H)$. In particular the Lagrangians of classes I and II in [2] satisfy $(\mathrm{H})$.

(ii) Lagrangians of the form

$$
L(x, u, z)=\frac{1}{2} \varphi(x, u)|z|^{2}+F(u)
$$

where $\varphi: \Omega \times \mathbb{R} \rightarrow \mathbb{R}$, satisfy $(\mathrm{H})$.

Recall the definition of the Euler-Lagrange derivative, formula (1). 
Theorem 3. Let $\Omega$ be a bounded domain of $\mathbb{R}^{N}, L \in \mathrm{C}^{2}\left(\Omega \times \mathbb{R} \times \mathbb{R}^{N}\right)$ a Lagrangian satisfying $(H)$ and $u \in \mathrm{C}^{2}(\Omega) \cap \mathrm{C}^{1}(\bar{\Omega})$ a non-trivial classical solution of Noether's equations

$$
\operatorname{div} T(x, u, D u)+L_{x}(x, u, D u)=0 .
$$

Then $u$ is a solution of the Euler-Lagrange equation

$$
\frac{\partial}{\partial x_{j}} L_{u, j}-L_{u}=0
$$

in $\Omega$.

Remark 5. By (8), equation (19) is equivalently written in the form

$$
\left(\frac{\partial}{\partial x_{j}} L_{u, j}-L_{u}\right) u_{, i}=0
$$

or in index-free notation

$$
\delta L(u) \cdot D u=0 .
$$

Note that (19) is a second order system of partial differential equations in $u$ and (20) is a single second order partial differential equation in $u$.

Proof. The proof begins exactly as the proof of Theorem 1 up to Step 3 where the proof of existence of the curve $\gamma$ is complete, with the obvious modification

$$
\mathrm{D}:=\{x \in \Omega: \delta L(u)(x)=0\} .
$$

For fixed $x_{0} \in \AA_{0}$ let $u\left(x_{0}\right)=: c_{0}$. Further let $f:=L_{u}$. Since $x_{0}$ and $x_{1}$ belong to the same connected component of $\mathrm{A}_{0}$, we have $u\left(x_{1}\right)=c_{0}$ and by $(\mathrm{H})$

$$
f\left(x_{0}, u\left(x_{0}\right), 0\right)=f\left(x_{1}, u\left(x_{0}\right), 0\right)=f\left(x_{0}, c_{0}, 0\right)=: d_{0} .
$$

We have

$$
\begin{aligned}
\left.\frac{\partial}{\partial x_{i}} L_{z_{i}}(x, u, D u)\right|_{x_{0}}= & L_{x_{i} z_{i}}\left(x_{0}, c_{0}, 0\right)+L_{u z_{i}}\left(x_{0}, c_{0}, 0\right) u_{, i}\left(x_{0}\right)+ \\
& L_{z_{i} z_{j}}\left(x_{0}, c_{0}, 0\right) u_{, i j}\left(x_{0}\right) \\
= & L_{x_{i} z_{i}}\left(x_{0}, c_{0}, 0\right)=0
\end{aligned}
$$

by $(\mathrm{H})$. Since by $14 \mathrm{f} x_{1} \in \mathrm{D}$, we have in a similar fashion

$$
\begin{aligned}
\left.\frac{\partial}{\partial x_{i}} L_{z_{i}}(x, u, D u)\right|_{x_{1}}= & L_{x_{i} z_{i}}\left(x_{1}, c_{0}, 0\right)+L_{u z_{i}}\left(x_{1}, c_{0}, 0\right) u_{, i}\left(x_{1}\right)+ \\
& L_{z_{i} z_{j}}\left(x_{1}, c_{0}, 0\right) u_{, i j}\left(x_{1}\right) \\
= & L_{x_{i} z_{i}}\left(x_{1}, c_{0}, 0\right)=f\left(x_{1}, c_{0}, 0\right)=d_{0}
\end{aligned}
$$

where the second equality from the end follows from the Euler-Lagrange equations:

$$
\left.\frac{\partial}{\partial x_{i}} L_{z_{i}}(x, u, D u)\right|_{x_{1}}=L_{u}\left(x_{1}, u\left(x_{1}\right), D u\left(x_{1}\right)\right)=f\left(x_{1}, c_{0}, 0\right) .
$$

Note that we have omitted a step involving the limiting process $t \rightarrow 1-$ along $\gamma$ and the associated continuity argument analogous to that applied in the proof of 
the following equation. From the first of $(\mathrm{H})$ and $\gamma(t) \in \stackrel{\circ}{\mathrm{A}}_{0}$ for all $t \in[0,1[$ we obtain

$$
\begin{aligned}
L_{x_{i} z_{i}}\left(x_{1}, c_{0}, 0\right) & =L_{x_{i} z_{i}}\left(\lim _{t \rightarrow 1-} \gamma(t), u\left(\lim _{t \rightarrow 1-} \gamma(t)\right), D u\left(\lim _{t \rightarrow 1-} \gamma(t)\right)\right) \\
& =\lim _{t \rightarrow 1-} L_{x_{i} z_{i}}(\gamma(t), u(\gamma(t)), D u(\gamma(t))) \\
& =\lim _{t \rightarrow 1-} L_{x_{i} z_{i}}\left(\gamma(t), c_{0}, 0\right)=0 .
\end{aligned}
$$

Combination of this equation with (24) yields $d_{0}=0$, hence by (22)

$$
f\left(x_{0}, c_{0}, 0\right)=0 \text {. }
$$

This means in particular

$$
\left.\frac{\partial}{\partial x_{i}} L_{z_{i}}(x, u, D u)\right|_{x_{0}}-f\left(x_{0}, u\left(x_{0}\right), D u\left(x_{0}\right)\right)=L_{x_{i} z_{i}}\left(x_{0}, c_{0}, 0\right)-f\left(x_{0}, c_{0}, 0\right)=0 .
$$

With this we have proved $\stackrel{\circ}{0}_{0} \subset \mathrm{D}$ and by (14) $\mathrm{A}_{0} \subset \mathrm{D}$.

Corollaries 1 and 2 transfer to the general Lagrangians conforming to $(H)$, with the obvious modifications:

Corollary 3. Under the assumptions of Theorem 1 if the set $\mathrm{A}_{0}:=\{x \in \Omega$ : $D u(x)=0\}$ has an interior point, then $L_{u}=0$ on all of $\AA_{0}$.

Corollary 4. Let $\Omega$ be a bounded domain of $\mathbb{R}^{N}$ and $L_{u}(x, u, z) \neq 0$ in $\Omega \times \mathbb{R} \times \mathbb{R}^{N}$. Then for every solution $u \in \mathrm{C}^{2}(\Omega) \cap \mathrm{C}^{1}(\bar{\Omega})$ of the partial differential equation in $u$

$$
\frac{\partial}{\partial x_{j}} L_{u, j}-L_{u}=0
$$

the set $\mathrm{A}_{0}:=\{x \in \Omega: D u(x)=0\}$ has no interior point.

Remark 4 holds as it is. Finally, we can state the equivalence of Euler-Lagrange and Noether equations as a general theorem by imposing conditions analogous to Theorem 2

Theorem 4. Let $\Omega$ be a bounded domain of $\mathbb{R}^{N}, g \in \mathrm{C}(\partial \Omega)$ a non-constant function and $\mathscr{H}:=\left\{u \in \mathrm{C}^{2}(\Omega) \cap \mathrm{C}^{1}(\bar{\Omega}): u \mid \partial \Omega=g\right\}$. Then the Euler-Lagrange and Noether equations for a Lagrangian satisfying $(H)$ are equivalent in $\mathscr{H}$.

Example 2. We consider the Lagrangian of p-Laplacian type [4]

$$
L(u, z)=\frac{1}{2} \varphi\left(|z|^{2}\right)+F(u)
$$

where $F \in \mathrm{C}(\mathbb{R})$ and $\varphi \in \mathrm{C}^{2}\left(\mathbb{R}^{+}\right)$such that $\varphi(0)=0$ and $\varphi^{\prime}(s) \geqslant 0 \forall s \geqslant 0$, which satisfies condition $(\mathrm{H})$. The energy-momentum tensor for this Lagrangian is given by

$$
T_{i j}=\varphi^{\prime}\left(|D u|^{2}\right) u_{, i} u_{, j}-\delta_{i j}\left(\frac{1}{2} \varphi\left(|D u|^{2}\right)+F(u)\right)
$$


By Theorem 4 the equations

$$
\operatorname{div}\left(\varphi^{\prime}\left(|D u|^{2}\right) D u \otimes D u\right)-D\left(\frac{1}{2} \varphi\left(|D u|^{2}\right)+F(u)\right)=0
$$

and

$$
\frac{\partial}{\partial x_{i}}\left(\varphi^{\prime}\left(|D u|^{2}\right) \frac{\partial u}{\partial x_{i}}\right)=F^{\prime}(u)
$$

with the boundary condition $u \mid \partial \Omega=g$, where $g \in \mathrm{C}(\partial \Omega)$ is a non-constant function, are equivalent.

\section{Applications}

5.1. Equivalence of admissible and inner variations. The $C^{2}$ solutions of EulerLagrange (respectively Noether) equations are critical (respectively inner critical) points of the corresponding variational functional. Since each inner variation gives rise to an admissible variation [6]

$$
w=D u \cdot h,
$$

the question arises if these two types of variation are equivalent. In this case, one could work with the smaller set of inner variations instead of the larger set of admissible variations. This might have an impact on the numerical computation of solutions. Next theorem gives an answer to this question.

Theorem 5. Let $J$ be a variational functional with Lagrangian $L \in \mathrm{C}^{2}(\Omega \times \mathbb{R} \times$ $\left.\mathbb{R}^{N}\right)$ satisfying $(H)$ and $u \in \mathrm{C}^{2}(\Omega) \cap \mathrm{C}^{1}(\bar{\Omega})$ a nontrivial function. Then the variational problem

$$
\delta J(u) v=0 \quad \forall v \in \mathscr{D}(\Omega)
$$

is equivalent to the problem

$$
\delta J(u) w=0 \quad \forall w \in \mathscr{I}(\Omega)
$$

where $\mathscr{I}(\Omega):=\{D u \cdot h: h$ is an inner variation of $u\}$. In simple words one can consider only inner variations of $u$ in Problem (25).

Proof. Equation (26) follows immediately from (25) when $u$ is $\mathrm{C}^{\infty}$. Otherwise $w \in \mathrm{C}_{c}^{1}(\Omega)$ while (25) requires $v \in \mathscr{D}(\Omega)$. In this case the proof follows by a simple density argument. For the converse, if (26) is valid, then $\delta J(u) D u \cdot h=0$ for all $h \in \mathscr{D}(\Omega)^{N}$. By the fundamental lemma of calculus of variations $\delta J(u) D u=0$, hence $u$ is a solution of Noether's equations and by Theorem 3 also a solution of the Euler-Lagrange equations. Since $u$ is $\mathrm{C}^{2}$, this means $u$ is a critical point of $J$, i.e. (25) is satisfied.

Remark 6. For $w=D u \cdot h, h \in \mathscr{D}(\Omega)^{N}$, we can prove by combining formulas (3), (6) and (8) that equation (26) may be written in the form $\mathfrak{d} J(u) h=0$ and recalling formula (2), $\left.\frac{d}{d t} J\left(u \circ \xi_{h}^{t}\right)\right|_{t=0}=0$. This admits of a possibly interesting interpretation: if one knows the distribution function of $u$, in order to solve the second order partial differential equation $\delta J(u)=0$, one has just to place the values of $u$ in their right position. 
5.2. Determination of Lagrangian from conservation law. As a second application, we present an example of "reverse engineering" the Lagrangian of a physical problem, mentioned in the Introduction. By this we mean there are experimental data available for a physical system and one would like to determine the system, in our case the Lagrangian. This is obviously a kind of inverse problem and, as it is well-known, such problems may have multiple solutions, or no solution at all and they may be not well-defined in certain senses, as for example the solution may not depend continuously on the data.

Assume there is a physical quantity $u$, which, as a conclusion from the examination of experimental data, is conserved according to the following physical law: the integral of the vector quantity $\mathbf{q}=\operatorname{div} \mathbf{T}$ over any set $V$ of three dimensional space remains constant and equal to 0 ; $\mathbf{T}$ being a tensor with components $T_{i j}=u_{, i} u_{, j}-\delta_{i j} \frac{1}{2}\left(|\nabla u|^{2}-\alpha u^{2}\right)$ in an orthogonal coordinate system and $\alpha$ a physical constant. In reality, this might be the diffusion of a rarefied substance contained in concentration $u$ in a medium, which is accompanied by degradation of the substance according to a first order reaction, or the conduction of heat in the presence of heat production or destruction at a rate proportional to the temperature $u$. The question is, if from these data one could determine a Lagrangian so that the Euler-Lagrange equations obtained from this Lagrangian coincide with the field equations deduced from experimental data.

Assuming that the system is confined in $\Omega$, from the conservation law obeyed by the field $u$ we obtain $\operatorname{div} \mathbf{T}(x)=0$ for any $x \in \Omega$. This is the experimentally determined field equations for $u$. If we assume that there is a Lagrangian $L=L(u, z)$ such that the energy-momentum tensor is $\mathbf{T}$, Theorem 1 guarantees that the corresponding Euler-Lagrange equations will be satisfied for every non-constant scalar field $u$ satisfying $\operatorname{div} \mathbf{T}(x)=0$. Thus, it remains to be checked if there is a Lagrangian having as energy-momentum tensor the tensor $\mathbf{T}$ given above. By formula (6) $L$ must satisfy the following first order system of partial differential equations

$$
z_{i} \frac{\partial L}{\partial z_{j}}-\delta_{i j} L=z_{i} z_{j}-\delta_{i j} \frac{1}{2}\left(|z|^{2}-\alpha u^{2}\right)
$$

for any $i, j \in\{1,2,3\}$. We note in passing that this is an overdetermined system, which may have no solutions. To solve (27) we observe that from

$$
z_{i} \frac{\partial L}{\partial z_{j}}=z_{i} z_{j}, \quad i \neq j
$$

we get

$$
L(u, z)=\frac{1}{2}|z|^{2}+F(u)
$$

where $F$ is a function to be determined from the additional equations

$$
z_{i} \frac{\partial L}{\partial z_{i}}-L=z_{i}^{2}-\frac{1}{2}\left(|z|^{2}-\alpha u^{2}\right)
$$


for $i \in\{1,2,3\}$; Einstein's summation convention does not apply in (29). From (29) on substituting $L$ by (28) we obtain $F(u)=-\frac{\alpha}{2} u^{2}$ and then

$$
L(u, z)=\frac{1}{2}|z|^{2}-\frac{\alpha}{2} u^{2},
$$

which is actually a solution of (27), as it may be directly verified. It follows from the way $L$ was determined, that (30) is a unique solution of (27).

\section{REFERENCES}

[1] Alikakos, N. Some basic facts on the system $\Delta u-W_{u}(u)=0$. Proc. Amer. Math. Soc. 139 (2011), no. 1, 153-162.

[2] Alikakos, N.; Faliagas, A. The stress-energy tensor and Pohozaev's identity for systems. Acta Math. Sci. 32 (2012), no. 1, 433-439.

[3] Bjorken, J. D.; Drell, S. D. Relativistic quantum fields. McGraw-Hill, 1964.

[4] Caffarelli, L.; Garofalo, N.; Segala, F. A gradient bound for entire solutions of quasi-linear equations and its consequences. Comm. Pure Appl. Math. 47 (1994), no. 11, 1457-1473.

[5] Dieudonné, J.-A. Treatise on Analysis. Volume I. Foundations of modern analysis. Enlarged and corrected printing. Academic, 1969.

[6] Faliagas, A. Construction of energy-momentum tensors in calculus of variations and applications to partial differential equations. Master's thesis. University of Athens, 2012. users.uoa.gr/ afaliaga/masters-thesis.pdf; dl.dropbox.com/u/56524894/masters-thesis.pdf.

[7] Feynman, R. P.; Leighton, R. B.; Sands, M. The Feynman lectures on physics. Vol. 1: Mainly mechanics, radiation, and heat. Addison-Wesley 1963.

[8] Giaquinta, M.; Hildebrandt, S. Calculus of Variations I: The Lagrangian Formalism. Grundlehren der mathematischen Wissenschaften. Springer, 2010.

[9] Noether, E. Invariant variation problems. English translation (2005). arxiv.org/pdf/physics/0503066v1.

Department of Mathematics, University of Athens, Panepistemiopolis, 15784 Athens, GREECE.

E-mail address: afaliaga@math.uoa.gr 